\title{
Evaluating the Value-Added of the Trabecular Bone Score in Patients with Rheumatoid Arthritis
}

\author{
Samaneh Tavassoli, MD $^{1 *}$; Alireza Rajaei, MD $^{2}$; Mohammad Mehdi Emam, MD²; Faraneh Farsad, MD $^{2}$ \\ 'Department of Internal Medicine, Sayyad Shirazi Hospital, Golestan University of Medical Sciences, Gorgan, Iran \\ 2Department of Internal Medicine, Shahid Beheshti University of Medical Sciences, Tehran, Iran
}

\begin{abstract}
Background: Rheumatoid arthritis (RA) presents with inflammation in the joints and bony tissues around them. The trabecular bone score (TBS) is a relatively new indicator that predicts fracture risk better than bone mineral density (BMD). The aim of the current study was to measure TBSs and BMD of patients with RA referring to Resalat Hospital, Tehran.

Methods: In this descriptive cross-sectional study, 129 men and women with RA entered the study through convenient sampling during 2016. TBS and BMD were measured in L1-L4. The relationships between age, sex, body mass index (BMI), duration of disease, and daily corticosteroids dose with TBS and BMD were determined by chi-square test, independent samples $t$ test, Pearson correlation, and linear and logistic regression.

Results: The TBS of $34.9 \%, 31.8 \%$, and $33.3 \%$ of study subjects were higher than $1.35,1.25-1.35$, and lower than 1.25 , respectively. The prevalence of TBS lower than 1.25 was $48.7 \%$ in women aged age more than 50 years. Age was the only predictor of low TBS in patients with RA. TBS and BMD were positively correlated in vertebral and hip bones. In women older than 50 years, BMI $(-1.292)$ and age (-1.330) were predictors of low TBS.

Conclusion: One-third of patients with RA were at risk of fracture. Gender and BMI were two factors which affected the TBS. This index can show the effect of disease on bones, which is related to age.

Keywords: Bone mineral density, Bone trabecular score, Osteoporosis, Rheumatoid arthritis

Cite this article as: Tavassoli S, Rajaei A, MM, Farsad F. Evaluating the value-added of the trabecular bone score in patients with rheumatoid arthritis. Arch Iran Med. 2021;24(3):193-198. doi: 10.34172/aim.2021.30.
\end{abstract}

Received: September 11, 2019, Accepted: October 18, 2020, ePublished: March 1, 2021

\section{Introduction}

Rheumatoid arthritis (RA) is the most prevalent inflammatory disease of the joint. ${ }^{1}$ Its skeletal complications include marginal and subchondral focal lesions, articular osteoporosis, reduced bone mass along with general bone loss. Patients with RA experience 3.9\% decrease in bone mineral density (BMD) in the lumbar spine and $2.5 \%$ in the femoral neck, respectively. ${ }^{2,3}$

The leading cause of osteoporosis and localized bone erosion is chronic inflammation of the synovial membranes. In addition, decreased bone mass is also associated with numerous well-known factors in RA such as the severity of disease activity, female gender, older age, glucocorticoid use, and reduced motility. These factors are independently and significantly related to disease progression. ${ }^{4-6}$ Also, decreased levels of total active vitamin $\mathrm{D}$ have been reported in RA patients, which is related to increased disease activity and musculoskeletal pain., ${ }^{7,8}$ Bone loss, bone erosion, and osteoporosis are seen in RA. ${ }^{9}$

Numerous studies have shown a common cellular pathway of the erosions seen in RA and osteoporosis, involving inflammatory activation of osteoclasts and decreased osteoblast activity. ${ }^{10}$

Decreased BMD in RA patients increases the risk of fracture and overall mortality, especially in women of the postmenopausal age. ${ }^{11}$ Measurement of loss of BMD by digital X-ray radiogrammetry in the hand is a quantitative method for early detection of osteoporosis by measuring the amount of minerals in the bone. ${ }^{12}$ Numerous studies have shown reduced bone density in $\mathrm{RA}^{13-17}$; however, mineral BMD alone is not a good predictor of the risk of bone fracture in these patients. ${ }^{18}$

Bone mineral densitometry is one of the diagnostic methods in which weak X-ray waves are used to determine bone density to measure the risk of fracture and osteoporosis. This index is more indicative of bone quantity and less indicative of bone quality. ${ }^{19-21}$

Bone quality can predict osteoporosis and bone fractures better than the quantity of minerals inside the bone. The trabecular score examines the bone microstructure and mostly represents bone quality. The bone microstructure is associated with bone mechanical strength and can predict bone fracture. For this reason, two bones with similar density may have different strengths. ${ }^{21,22}$

Loss of bone in osteoporosis is also associated with changes in the structure of the bone. The results of some studies have shown that this index can better indicate bone strength status. ${ }^{19,20,23}$ Few studies have been performed to 
determine the factors affecting the trabecular bone score (TBS) in patients with RA. The purpose of this study was to measure the TBS in patients with RA in Resalat Hospital, Tehran, in 2016.

\section{Materials and Methods}

In this descriptive cross-sectional study, TBS was determined in patients with RA in Resalat Hospital in Tehran in 2016. A minimum sample size of 87 individuals was calculated by selecting a standard deviation of 0.19 based on the study by Pothuaud et al, ${ }^{24}$ and $95 \%$ confidence level and 0.04 error. Finally, in this study, considering the probability of sample attrition, 129 patients with RA who referred to Resalat hospital in Tehran were selected and evaluated by sequential non-probability sampling.

Bone mineral densitometry was performed for all patients in the lumbar region of the pelvis using a Discovery W (S/N 83167) homologous device using digital X-ray radiogrammetry. Densitometry is reported as grams per $\mathrm{cm}^{3}$. The mean and standard deviation of the T-score are also reported and compared with a group of healthy 30-year-old individuals of the same sex.

A T-score greater than $(-1)$ is considered as normal, range of -1 and -2.5 as osteopenia and less than -2.5 as osteoporosis (in this case, bone density is 2.5 standard deviations lower than the lowest mean in a 30 -year-old man or woman). The TBS indicates the difference in gray levels in digital X-ray radiogrammetry. Low values of bone trabeculae indicate weak structure and higher values indicate strong structure.

In the present study, lumbar spine mineral densitometry in L1-L4 vertebrae was expressed as mean by removing fractured or deformed vertebrae (due to osteoarthritis). Patients' TBS was also analyzed in the L1-L4 lumbar vertebrae and all vertebrae removed in BMD examination were also removed in the TBS test.

TBS is classified as follows: Fully degraded microarchitecture: TBS equal to or less than 1200; Partially degraded microarchitecture: TBS between 1.200 and <1.350; and Normal microarchitecture: TBS more than 1.350 .
Statistical Analysis

The SPSS 16 software was used to analyze data using chisquare test, independent $t$ test, Pearson correlation, and linear and logistic regression (backward method). The significance level of all tests was considered to be less than 0.05 .

\section{Results}

In this study, 129 patients including 20 males (15.5\%) and 109 females $(84.5 \%)$ were enrolled. The mean \pm standard deviation (SD) of age was $56.33 \pm 13.46$ years. The mean \pm standard deviation of body mass index (BMI) was 27.87 $\pm 5.24\left(\mathrm{~kg} / \mathrm{m}^{2}\right)$.

Mean \pm SD of disease duration was $6.91 \pm 4.14$ years; $54.3 \%$ of patients did not take corticosteroids, $19.4 \%$ (25 patients) used less than $5 \mathrm{mg}$ corticosteroids daily, and $26.4 \%$ (34 patients) used more than $5 \mathrm{mg}$ daily.

Mean (SD) of BMD was $0.73(0.13)$ and $0.92(0.14)$ in the pelvis and lumbar spine, respectively. Mean TBS \pm SD was $1.30 \pm 0.11$. Also, $34.9 \%$ of patients had a trabecular index more than 1.35 , while for $31.8 \%$, it was between $1.25-1.35$ and for $33.3 \%$, it was less than 1.25 .

There was a significant and inverse correlation between age and pelvic-lumbar bone density and TBS $(P<0.05)$. There was a direct correlation between BMI and bone density in the lumbar area and a negative correlation with the TBS $(P<0.05)$.

There was also a significant and inverse correlation between duration of disease and pelvic and lumbar bone density and TBS $(P<0.05)$. The correlation between TBS and lumbar and pelvic density was statistically significant $(P<0.05)$ (Table 1$)$.

Pelvic bone density was significantly different between the males and females, with men having a higher score. The rest of the indices showed no significant difference between men and women, or between those taking less than $5 \mathrm{mg} /$ day of corticosterone and those taking more than $5 \mathrm{mg} /$ day. (Tables 2 and 3).

Table 4 presents the linear regression analysis of the three models associated with the three indices investigated. Linear regression analysis showed that age was a predictor

Table 1. Correlation between Age, Body Mass Index and Duration of Disease with Bone Density and Trabecular Bone Score

\begin{tabular}{|c|c|c|c|c|c|c|}
\hline & & Age & Body Mass Index & Duration of Disease & Pelvic Bone Density & Lumbar Bone Density \\
\hline \multirow{2}{*}{ Body mass index } & Correlation coefficient & -0.064 & & & & \\
\hline & $P$ value & 0.470 & & & & \\
\hline \multirow{2}{*}{ Duration of disease } & Correlation coefficient & 0.098 & -0.166 & & & \\
\hline & $P$ value & 0.450 & 0.201 & & & \\
\hline \multirow{2}{*}{ Pelvic bone density } & Correlation coefficient & $-0.461^{* *}$ & $0.222^{*}$ & $-0.318^{*}$ & & \\
\hline & $P$ value & $<0.001$ & 0.011 & 0.012 & & \\
\hline \multirow{2}{*}{ Lumbar bone density } & Correlation coefficient & $-0.324^{* *}$ & $0.277^{* *}$ & $-0.308^{*}$ & $0.671^{* *}$ & \\
\hline & $P$ value & $<0.001$ & 0.001 & 0.016 & $<0.001$ & \\
\hline \multirow{2}{*}{ Trabecular bone score } & Correlation coefficient & $-0.508^{* *}$ & $-0.295^{* *}$ & $-0.284^{*}$ & $0.465^{* *}$ & $0.521^{* *}$ \\
\hline & $P$ value & $<0.001$ & 0.001 & 0.027 & $<0.001$ & $<0.001$ \\
\hline
\end{tabular}


Table 2. Comparison of Gender Trabecular Bone Score, Lumbar Spine and Pelvic Density Base on Gender

\begin{tabular}{lccc}
\hline & \multicolumn{2}{c}{ Gender } & \multirow{2}{*}{ Mean Difference (95 \% Cl) } \\
\cline { 2 - 3 } & Male & Female & $0.031(-0.024,0.087)$ \\
\hline Trabecular bone score & $1.33 \pm 0.09$ & $1.30 \pm 0.12$ & $0.076(0.011,0.140)$ \\
Pelvic bone density & $0.79 \pm 0.11$ & $0.72 \pm 0.13$ & 0.27 \\
Lumbar bone density & $0.93 \pm 0.14$ & $0.92 \pm 0.14$ & $0.014(-0.057,0.085)$ \\
\hline
\end{tabular}

${ }^{\mathrm{a}}$ Independent $t$ test.

Table 3. Comparison of Gender Trabecular Bone Score, Lumbar Spine and Pelvic Density Based on Daily Corticosteroid Usage

\begin{tabular}{|c|c|c|c|c|}
\hline & \multicolumn{2}{|c|}{ Daily Corticosteroid Usage } & \multirow{2}{*}{ Mean Difference $(95 \% \mathrm{Cl})$} & \multirow{2}{*}{$P$ Value $^{\mathrm{a}}$} \\
\hline & $>5 \mathrm{mg} / \mathrm{d}$ & $<5 \mathrm{mg} / \mathrm{d}$ & & \\
\hline Trabecular bone score & $1.30 \pm 0.11$ & $1.30 \pm 0.12$ & $-0.0003(-0.046,0.045)$ & 0.988 \\
\hline Pelvic bone density & $0.72 \pm 0.13$ & $0.73 \pm 0.13$ & $-0.007(-0.061,0.046)$ & 0.784 \\
\hline Lumbar bone density & $0.92 \pm 0.15$ & $0.93 \pm 0.13$ & $0.009(-0.049,0.068)$ & 0.757 \\
\hline
\end{tabular}

a Independent $t$ test.

Table 4. Linear Regression of Bone Trabecular Score Predictors

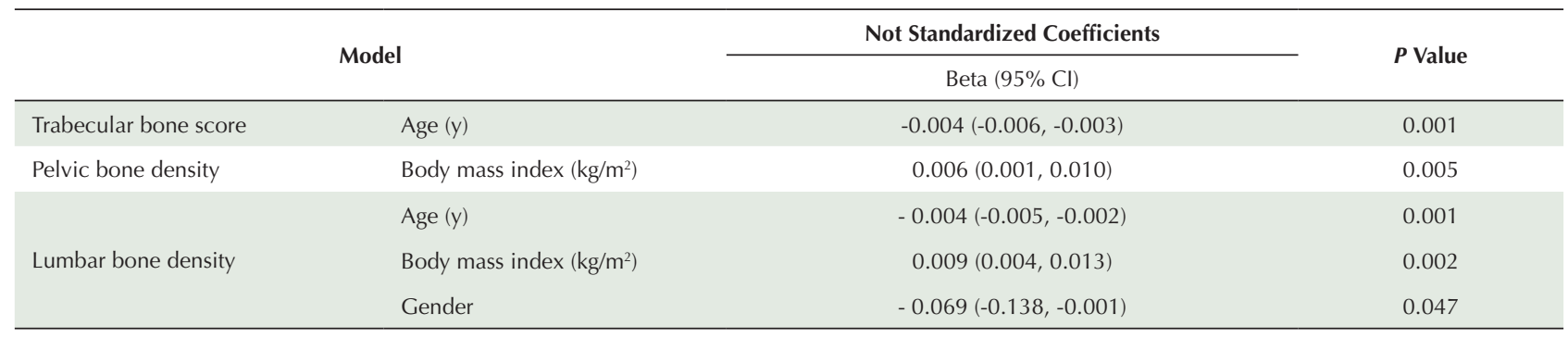

of decreased TBS. Thus, with each year increase in age, the TBS decreased by $0.04\left(\mathrm{R}^{2}=0.266\right)$.

Regarding pelvic bone density, BMI was predictive of BMD, and by increasing one unit in BMI, 0.009 was added to bone density $\left(\mathrm{R}^{2}=0.131\right)$. With each year increase in age, lumbar BMI decreased by 0.004 , with increasing one unit in BMI, a 0.009 increase was shown in lumbar BMD, and women had 0.132 lower lumbar BMD than men $\left(\mathrm{R}^{2}=0.370\right)$.

According to the results of Table 5, there was an association between TBS and bone density, such that with increasing bone density, the TBS also increased. Logistic regression was performed by placing the subjects in two groups of TBS $<1.25$ and greater than 1.25 . The results showed that the risk of fractures increased by 1.12 times with each year increase in age and the risk of fracture increased by 1.31 times with each unit increase in BMI (Table 6).

Using statistical tests on women older than 50 years as the index group, the results showed that the risk of fracture increased 1.29 times with one unit increase in BMI, and 1.33 times with each year increase in the duration of disease.

\section{Discussion}

According to the results of this study on RA patients in Resalat Hospital in Tehran in 2016, a high percentage of RA patients were at high risk of osteoporosis and bone fracture and more than one-third of RA patients were at risk for pathologic fractures. By setting a TBS of less than

Table 5. Relationship of Bone Density T Score with Trabecular Bone Score

\begin{tabular}{|c|c|c|c|c|c|}
\hline & & \multicolumn{3}{|c|}{ Bone Density T Score } & \multirow{2}{*}{$P$ Value } \\
\hline & & $>(-1)$ & ( -1 to -2.5$)$ & $>(-2.5)$ & \\
\hline \multirow{3}{*}{ TBS } & TBS $\leq 1.200$ & $7(14 \%)$ & $27(49.1 \%)$ & $11(45.8)$ & \\
\hline & TBS $>1.200$ and $<1.350$ & $14(28 \%)$ & $16(29.1 \%)$ & $11(45.8 \%)$ & $\chi^{2}=27.914, \mathrm{df}=4, P<0.001$ \\
\hline & TBS $>1.350$ & $29(58 \%)$ & $12(21.8 \%)$ & $2(8.3 \%)$ & \\
\hline
\end{tabular}

TBS, trabecular bone score. 
Table 6. Logistic Regression of Factors Associated With Fracture Risk

\begin{tabular}{lccc}
\hline & Odds Ratio & CI & P Value \\
\hline Gender & 2.060 & $(0.174,24.456)$ & 0.567 \\
Age $(\mathrm{y})$ & 1.128 & $(1.036,1.229)$ & 0.006 \\
Body mass index $\left(\mathrm{kg} / \mathrm{m}^{2}\right)$ & 1.319 & $(1.074,1.618)$ & 0.008 \\
\hline Dosage of corticosteroid $(\mathrm{mg})$ & 0.565 & $(0.122,2.613)$ & 0.465 \\
Duration of disease (year) & 4.506 & $(0.834,24.337)$ & 0.080 \\
\hline
\end{tabular}

1.25 as the cutoff value for fracture risk, the results showed that elevated BMI and duration of RA were predictors of increased risk of fracture.

The TBS is a newer parameter that examines bone microstructure quality while bone density measures the amount of minerals present in bone. The results showed that this parameter indicated the possibility of predicting bone strength in the face of blows and is a more accurate index.

RA has been shown to affect bone density. In this study, we showed that more than one-third of people with RA had a trabecular score of less than 1.25 , and this percentage was about half of the sample in women over 50 years of age. In women over 50 , the risk of fracture increased with increasing BMI and duration of disease. Previous research has also shown that RA can alter bone structure, ${ }^{25,26}$ and an association has been shown between BMI and osteoporosis. ${ }^{27-30}$ However, previous studies have suggested BMI as a protective factor against the decrease in BMD. ${ }^{31,32}$

The results of this study showed that menopausal women with RA are more likely to have decreased bone quality with increased BMI. Longer duration of the disease was also associated with increased risk of fracture in postmenopausal women - a result that has been considered in previous studies. This is a conclusion that needs further investigation.

In the present study, no association was seen between gender and increased risk of fracture, and gender did not predict a decrease in TBS. Studies have shown that postmenopausal women are at higher risk for osteoporosis. ${ }^{33,34}$ In the present study, the probability of being in the high-risk group for fractures in women was close to $50 \%$, while in the total sample, it was slightly over $30 \%$, indicating that menopausal women are more likely to have fractures. Increasing age was a predictor of increased risk of fracture, which has been confirmed in previous studies. ${ }^{35-37}$

The duration of illness in postmenopausal women was one of the predictors of a higher risk of fracture.

Like other studies, our research also had some limitations; one of the critical limitations of the present study was its cross-sectional nature, which makes it impossible to evaluate reverse causality and the consequences of decreased TBS in the development of true fracture. It is recommended that cohort studies should be performed on these patients with follow-up of actual fractures as well as changes in TBS and BMD.

The small sample size in some subgroups, suggesting a possibility of sparse-data bias, should also be considered as a limitation of our study.

Another limitation of the study is the criterion of risk. Bone trabecular score under 1.25 has been established in postmenopausal women, while no criteria are available for other groups; therefore, no proper comparison can be made between the sexes or between postmenopausal and non-menopausal women. Future studies with large sample sizes on patients of both sexes and women of reproductive age are recommended. TBS helps diagnose cases requiring treatment or at least more careful follow-up. TBS is also valuable in obese and older patients with spinal cord changes due to degenerative joint disease and is very helpful in identifying those who are at risk of fractures.

In conclusion, in this study, increasing BMI and duration of RA were related to the increased risk of bone fractures due to osteoporosis. So, it could be concluded that special attention should be paid to higher weight women with RA with a more extended period. Overall, age predicts a decrease in the TBS; so, people with RA need more attention and interventions should be developed to reduce the effects of the disease, especially on the bones.

\section{Authors' Contribution}

ST participated in acquisition of data, analysis and interpretation, writing and discuss in result, drafted or provided critical revision of the article and final approval and agreed to be accountable for all aspect of the word. MME and FF participated in reading and providing, drafted critical revision of the article, final approval and agreed to be accountable for all aspect of the word. AR contributed to disign of the article.

\section{Conflict of Interest Disclosures \\ None.}

\section{Ethical Statement}

This study was approyed by Shahid Beheshti Medical science ethical committee with No. IR.SBMU.MST.IEC.1395.295.

\section{References}

1. Wheater G, Elshahaly M, Naraghi K, Tuck SP, Datta HK, van Laar JM. Changes in bone density and bone turnover in patients with rheumatoid arthritis treated with rituximab, results from an exploratory, prospective study. PLoS One. 2018;13(8):e0201527. doi: 10.1371/journal.pone.0201527.

2. Franck $\mathrm{H}$, Gottwalt J. Peripheral bone density in patients with rheumatoid arthritis. Clin Rheumatol. 2009;28(10):1141-5. doi: 10.1007/s10067-009-1211-2.

3. Juhasz B, Gulyas K, Horvath A, Petho Z, Bhattoa HP, Vancsa A, et al. Comparison of peripheral quantitative computed tomography forearm bone density versus DXA in rheumatoid arthritis patients and controls. Osteoporos Int. 2017;28(4):1271-7. doi: 10.1007/s00198-016-3850-x.

4. Haugeberg G, Morton S, Emery P, Conaghan PG. Effect of intra-articular corticosteroid injections and inflammation on periarticular and generalised bone loss in early rheumatoid arthritis. Ann Rheum Dis. 2011;70(1):184-7. doi: 10.1136/ ard.2009.128124. 
5. Roldan JF, Del Rincon I, Escalante A. Loss of cortical bone from the metacarpal diaphysis in patients with rheumatoid arthritis: independent effects of systemic inflammation and glucocorticoids. J Rheumatol. 2006;33(3):508-16.

6. Schett G. Rheumatoid arthritis: inflammation and bone loss. Wien Med Wochenschr. 2006;156(1-2):34-41. doi: 10.1007/ s10354-005-0244-7

7. Alwan A, Al Rassy N, Berro AJ, Rizkallah M, Matta J, Frenn F, et al. Vitamin D and Trabecular Bone Score in a Group of Young Lebanese Adults. J Clin Densitom. 2018;21(3):453-8. doi: 10.1016/j.jocd.2018.02.002.

8. Goldman AL, Donlon CM, Cook NR, Manson JE, Buring JE, Copeland T, et al. VITamin D and OmegA-3 TriaL (VITAL) bone health ancillary study: clinical factors associated with trabecular bone score in women and men. Osteoporos Int. 2018;29(11):2505-15. doi: 10.1007/s00198-018-4633-3.

9. Rossini M, Bagnato G, Frediani B, lagnocco A, G LAM, Minisola G, et al. Relationship of focal erosions, bone mineral density, and parathyroid hormone in rheumatoid arthritis. J Rheumatol. 2011;38(6):997-1002. doi: 10.3899/ jrheum.100829

10. Orsolini G, Caimmi C, Viapiana O, Idolazzi L, Fracassi E, Gatti $D$, et al. Titer-dependent effect of anti-citrullinated protein antibodies on systemic bone mass in rheumatoid arthritis patients. Calcif Tissue Int. 2017;101(1):17-23. doi: 10.1007/ s00223-017-0253-8.

11. Book C, Algulin J, Nilsson JA, Saxne T, Jacobsson L. Bone mineral density in the hand as a predictor for mortality in patients with rheumatoid arthritis. Rheumatology (Oxford). 2009;48(9):1088-91. doi: 10.1093/rheumatology/kep169.

12. Kapetanovic MC, Lindqvist E, Algulin J, Jonsson K, Saxne $\mathrm{T}$, Eberhardt $\mathrm{K}$, et al. Early changes in bone mineral density measured by digital X-ray radiogrammetry predict up to 20 years radiological outcome in rheumatoid arthritis. Arthritis Res Ther. 2011;13(1):R31. doi: 10.1186/ar3259.

13. Eekman DA, Vis M, Bultink IE, Kuik DJ, Voskuyl AE, Dijkmans $\mathrm{BA}$, et al. Stable bone mineral density in lumbar spine and hip in contrast to bone loss in the hands during long-term treatment with infliximab in patients with rheumatoid arthritis. Ann Rheum Dis. 2011;70(2):389-90. doi: 10.1136/ ard.2009.127787.

14. Krieckaert CL, Nurmohamed MT, Wolbink G, Lems WF. Changes in bone mineral density during long-term treatment with adalimumab in patients with rheumatoid arthritis: a cohort study. Rheumatology (Oxford). 2013;52(3):547-53. doi: $\quad$ 10.1093/rheumatology/kes320.

15. Hoff M, Kvien TK, Kalvesten J, Elden A, Haugeberg G. Adalimumab therapy reduces hand bone loss in early rheumatoid arthritis: explorative analyses from the PREMIER study. Ann Rheum Dis. 2009;68(7):1171-6. doi: 10.1136/ ard.2008.091264.

16. Hoff M, Haugeberg G, Odegard S, Syversen S, Landewe R, van der Heijde D, et al. Cortical hand bone loss after 1 year in early rheumatoid arthritis predicts radiographic hand joint damage at 5-year and 10-year follow-up. Ann Rheum Dis. 2009;68(3):324-9. doi: 10.1136/ard.2007.085985.

17. Rezaei H, Saevarsdottir S, Geborek P, Petersson IF, van Vollenhoven RF, Forslind K. Evaluation of hand bone loss by digital X-ray radiogrammetry as a complement to clinical and radiographic assessment in early rheumatoid arthritis: results from the SWEFOT trial. BMC Musculoskelet Disord. 2013;14:79. doi: 10.1186/1471-2474-14-79.

18. Aeberli D, Eser P, Bonel H, Widmer J, Caliezi G, Varisco PA, et al. Reduced trabecular bone mineral density and cortical thickness accompanied by increased outer bone circumference in metacarpal bone of rheumatoid arthritis patients: a crosssectional study. Arthritis Res Ther. 2010;12(3):R119. doi: 10.1186/ar3056.
19. Ripamonti C, Lisi L, Buffa A, Gnudi S, Caudarella R. The trabecular bone score predicts spine fragility fractures in postmenopausal caucasian women without osteoporosis independently of bone mineral density. Med Arch. 2018;72(1):46-50. doi: 10.5455/medarh.2018.72.46-50.

20. Watanabe R, Tai N, Hirano J, Ban Y, Inoue D, Okazaki $R$. Independent association of bone mineral density and trabecular bone score to vertebral fracture in male subjects with chronic obstructive pulmonary disease. Osteoporos Int. 2018;29(3):615-23. doi: 10.1007/s00198-017-4314-7.

21. Iglesias SG, Dominguez MLM, Herrero EF, Martinez-Pueyo JI, Arroba CM, Diaz-Guerra GM, et al. Trabecular bone score and bone mineral density in patients with postsurgical hypoparathyroidism after total thyroidectomy for differentiated thyroid carcinoma. Surgery. 2019;165(4):814-819. doi: 10.1016/j.surg.2018.10.034.

22. Rianon N, Ambrose CG, Buni M, Watt G, Reyes-Ortiz C, Lee $M$, et al. Trabecular bone score is a valuable addition to bone mineral density for bone quality assessment in older Mexican American women with type 2 diabetes. J Clin Densitom. 2018;21(3):355-9. doi: 10.1016/j.jocd.2018.02.004.

23. Aleksova J, Kurniawan S, Elder GJ. The trabecular bone score is associated with bone mineral density, markers of bone turnover and prevalent fracture in patients with end stage kidney disease. Osteoporos Int. 2018;29(6):1447-55. doi: 10.1007/s00198-018-4468-y.

24. Pothuaud L, Barthe N, Krieg MA, Mehsen N, Carceller P, Hans D. Evaluation of the potential use of trabecular bone score to complement bone mineral density in the diagnosis of osteoporosis: a preliminary spine BMD-matched, case-control study. J Clin Densitom. 2009;12(2):170-6. doi: 10.1016/j. jocd.2008.11.006.

25. Ruaro B, Casabella A, Paolino S, Pizzorni C, Ghio M, Seriolo C, et al. Dickkopf-1 (Dkk-1) serum levels in systemic sclerosis and rheumatoid arthritis patients: correlation with the trabecular bone score (TBS). Clin Rheumatol. 2018;37(11):3057-62. doi: 10.1007/s10067-018-4322-9.

26. Killinger Z, Gajdarova L, Kuzma M, Krajcovicova A, Brazdilova $\mathrm{K}$, Jackuliak $\mathrm{P}$, et al. Biologic treatment in comparison to methotrexate has positive effect on trabecular bone score in rheumatoid arthritis patients: 1-year follow-up. Acta Clin Belg. 2019;74(2):121-5. doi: 10.1080/17843286.2018.1512189.

27. Jiang $X$, Good LE, Spinka R, Schnatz PF. Osteoporosis screening in postmenopausal women aged 50-64 years: BMI alone compared with current screening tools. Maturitas. 2016;83:59-64. doi: 10.1016/j.maturitas.2015.09.009.

28. Li Y, Hui M, Chang X, Li M, Wang Y, Zhang B, et al. BMl reduction and vitamin $D$ insufficiency mediated osteoporosis and fragility fractures in patients at nutritional risk: a crosssectional study. Eur J Clin Nutr. 2018;72(3):455-9. doi: 10.1038/s41430-017-0067-9.

29. Rithirangsriroj K, Panyakhamlerd K, Chaikittisilpa S, Chaiwatanarat T, Taechakraichana N. Osteoporosis in different age-groups and various body mass index (BMI) ranges in women undergoing bone mass measurement at King Chulalongkorn Memorial Hospital. J Med Assoc Thai. 2012;95(5):644-9.

30. Skrzek A, Koziel S, Ignasiak Z. The optimal value of BMI for the lowest risk of osteoporosis in postmenopausal women aged 40-88 years. Homo. 2014;65(3):232-9. doi: 10.1016/j. jchb.2014.01.003.

31. Glogowska-Szelag J. Assessment of the relationship between bmd and body mass index bmi in women with postmenopausal osteoporosis [ in Polish]. Wiad Lek. 2018;71(9):1714-8.

32. Huang Y, Chen N, Miao D. Effect and mechanism of pyrroloquinolinequinone on anti-osteoporosis in Bmi-1 knockoutmice-Anti-oxidant effect of pyrroloquinolinequinone. Am J Transl Res. 2017;9(10):4361-74. 
33. Beresheim AC, Pfeiffer SK, Grynpas MD, Alblas A. Sex-specific patterns in cortical and trabecular bone microstructure in the Kirsten Skeletal Collection, South Africa. Am J Hum Biol. 2018;30(3):e23108. doi: 10.1002/ajhb.23108.

34. Wang L, Cheng XG, Su YB, Brown K, Xu L, Li K, et al. Sexrelated variations in cortical and trabecular bone of the femoral neck in an elderly Chinese population. Osteoporos Int. 2017;28(8):2391-9. doi: 10.1007/s00198-017-4043-y.

35. Torgutalp SS, Babayeva N, Kara OS, Ozkan O, Donmez G, Korkusuz F. Trabecular bone score of postmenopausal women is positively correlated with bone mineral density and negatively correlated with age and body mass index. Menopause. 2019;26(10):1166-70. doi: 10.1097/ GME.0000000000001375.

36. Guagnelli MA, Winzenrieth R, Lopez-Gonzalez D, McClung MR, Del Rio L, Clark P. Bone age as a correction factor for the analysis of trabecular bone score (TBS) in children. Arch Osteoporos. 2019;14(1):26. doi: 10.1007/s11657-019-05736.

37. Iki M, Tamaki J, Sato Y, Winzenrieth R, Kagamimori S, Kagawa $\mathrm{Y}$, et al. Correction to: Age-related normative values of trabecular bone score (TBS) for Japanese women: the Japanese Population-based Osteoporosis (JPOS) study. Osteoporos Int. 2019;30(1):253-5. doi: 10.1007/s00198-018-4779-z.

(c) (1) (C) 2021 The Author(s). This is an open-access article distributed under the terms of the Creative Commons Attribution License (http://creativecommons. org/licenses/by/4.0), which permits unrestricted use, distribution, and reproduction in any medium, provided the original work is properly cited. 